\title{
Home based unsupervised rehabilitation protocol following rotator cuff repair has good outcomes: A prospective study
}

\author{
Rohan A Habbu ${ }^{1}$
}

\section{Abstract}

Background: Rehabilitation after rotator cuff repair achieves good results but it involves time and costs. A simple home based program will reduce costs, time to travel and chance of exposure in a pandemic scenario. The objective of the study was to assess outcomes of home based rehabilitation protocol following rotator cuff repair.

Methods: This was a prospective study of 42 patients who underwent rotator cuff repair followed by an unsupervised home based rehabilitation protocol. The tears were identified using clinical examination and Magnetic Resonance Imaging. The cuff repair was done with mini open incision technique. The patients were taught an exercise protocol, which they did by themselves at home. There was no daily supervision by the treating surgeon or a physiotherapist. The study measured pain scores, return to work, range of motion and failures at one year.

Results: Mean duration of followup was 14 months. Visual Analogue Score for pain improved from preoperative mean 7.5 to followup mean 1.3. Disability of the Arm, Hand and Shoulder score, measured in 26 patients improved from preoperative mean 33.2 to followup mean 4.5. Range of motion improved in 39 patients. Cuff strength was normal in 39 patients. 37 patients returned to full work by three months. There were three failures with one patient developing significant stiffness and two patients showing a retear. The retear was seen in patients with large retracted tears and the repair was done under tension.

Conclusion: Home based exercise protocol for postoperative rehabilitation in rotator cuff repairs can achieve good results in carefully selected patients. The study does not recommend this for large tears with difficult repairs. Since the patients are doing the postoperative rehabilitation at home, this reduces the costs, time lost in travel and exposure to external factors.

Keywords: Rotator cuff, mini open repair, unsupervised rehabilitation, motion

\section{Introduction}

Rotator cuff tears are frequently seen in the elderly as well as in athletic population. Degenerative tears are more common in elderly patients while traumatic tears are common in young or athlete patients. These tears are routinely treated with either arthroscopic repairs or miniopen repair techniques, both of which have good results. [1, 2] An important aspect of treatment of such tears is the postoperative rehabilitation. [3] Supervised physiotherapy is

${ }^{1}$ Department of Upper Limb Surgery, Prime Health Clinic Ground floor, Ram Janki Apts Subhash Road, Vile Parle East, Mumbai 400057. India

Address of Correspondence

Dr. Rohan A Habbu,

Department of Upper Limb Surgery, Prime Health Clinic Ground floor,

Ram Janki Apts Subhash Road, Vile Parle East, Mumbai 400057. India

E-mail: dr_habbu@yahoo.com routinely advised, in which the patients visit the therapist or the therapist visits the home. A video based supervised therapy has also been shown to have good results. [4] However, physical or virtual involvement of a therapist can increase the costs of the treatment. $[4,5]$ Time is also lost in completing the travel and the therapy sittings. In the current pandemic scenario, there is also an increased risk of exposure. On the other hand, if the patient is left to do unsupervised rehabilitation, the repair may be put at risk. [6] The advantage of a simple home-based rehabilitation program done by patients themselves can cut down on costs, time and exposure to external factors. An ideal home based rehabilitation protocol for rotator cuff repairs should allow no stress on the repair and at the same time maintain the range of motion. The objective of the present study is to assess the outcomes of a simple home-based rehabilitation protocol performed by patients themselves at home.

\section{Materials \& Methods}

This is a prospective study done between 2015 to 2017. The study included patients who underwent rotator cuff repair through a miniopen incision. A rotator cuff tear was defined as full thickness complete tear of the supraspinatus, infraspinatus or both. This diagnosis was based on clinical

Submitted Date: 30-Apr-21, Review Date: 21-May-21, Accepted Date: 28-May-21 \& Published Date: 31-Dec-21

Journal of Clinical Orthopaedics | Available on www.jcorth.com | DOI:10.13107/jcorth.2021.v06i02.443 This is an Open Access article distributed under the terms of the Creative Commons Attribution NonCommercial-Share Alike 4.0 License (http://creativecommons.org/licenses/by-nc-sa/4.0) which allows others to remix, tweak, and build upon the work non-commercially as long as appropriate credit is given and the new creation are licensed under the identical terms. 


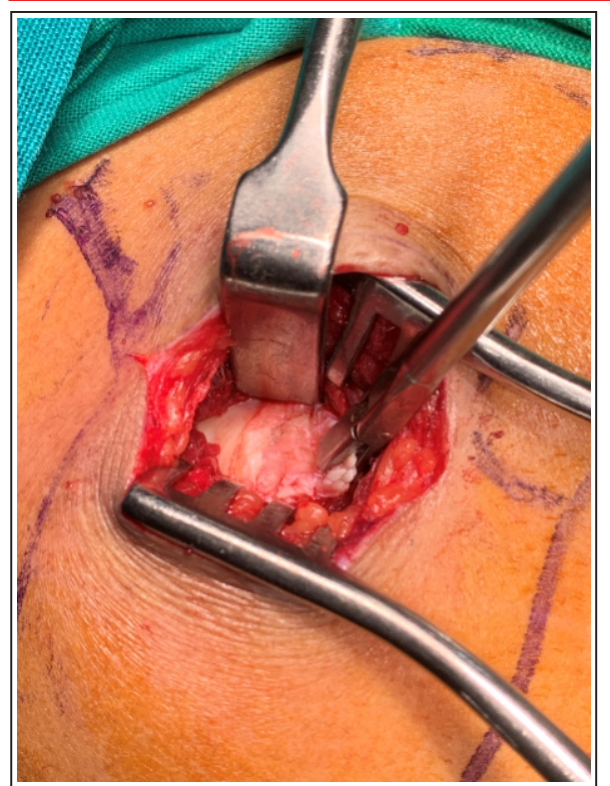

Figure 1: Cuff tear.

The rotator cuff tear was identified and mobilized to bring the edge to the foot print

examination and magnetic resonance imaging (MRI). We excluded patients who had partial cuff tears, additional concomitant surgeries or had previous shoulder surgeries.

During the study period, 62 patients underwent rotator cuff repair surgery. Of these, 42 patients satisfied the inclusion criteria and were included in the present study. There were 19 males and 23 females. Mean age of the patients was 60 years (range, 49 to 74). Dominant side was affected in 19 patients. Four patients were involved in heavy occupation (construction, farming). The other 38 had sedentary lifestyle. Mean duration of symptoms was 3.5 months (range, 1 to 9). Mode of injury included blunt trauma (11), standing height fall (21), fall from height (1) and no cause in nine patients. The chief complaint in all 42 patients was pain. Rotator cuff was found weak in all 42 patients when tested against resistance. All 42 patients showed some restriction of motion when compared to opposite side. Radiographs were reported as normal in 39 patients. Three patients were reported to have proximal migration of humeral head. MRI showed full thickness tear of supraspinatus in 40 patients. Two patients had tear of both

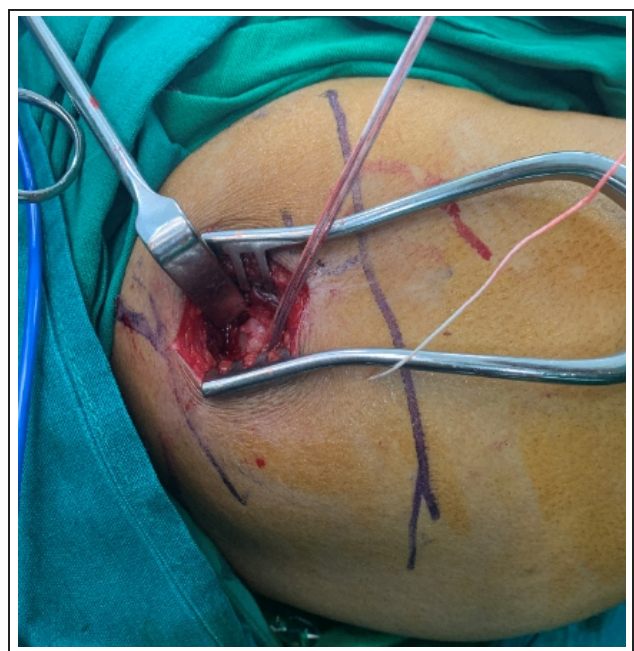

Figure 2: Cuff repair.

The rotator cuff tear was repaired using one double loaded anchor with mattress pattern stitches.

supraspinatus and infraspinatus. There was no patient with isolated infraspinatus tear. Cuff edge retraction on MRI was reported as upto two $\mathrm{cm}$ in 32 patients and more than two $\mathrm{cm}$ was seen in six patients. There was no retraction in four patients.

\section{Surgical technique}

The surgery was performed under general anaesthesia. A vertical incision measuring three $\mathrm{cm}$ was made over the anterolateral aspect of the shoulder. The incision began just distal to the anterolateral corner of the acromion. Blunt dissection was carried out to identify the raphe between the anterior third and middle third parts of the deltoid muscle. This plane was developed bluntly and the muscles edges were gently retracted. Subacromial bursa and subdeltoid bursa were excised. The tear in the rotator cuff tendons was identified and mobilized. (Fig 1) The footprint and the tendon edges were prepared. The cuff was then repaired in a single row configuration with one or two $5.5 \mathrm{~mm}$ double loaded titanium anchors. (Fig 2) The decision of number of anchors was made on table depending on the tear configuration. A mattress pattern of stitch was taken through the good tendon tissue and the threads were tied down with shoulder in neutral rotation. A single anchor was used in eight patients while two anchors were used in 34 patients. The stability of the repair was tested by putting the shoulder through a gentle range of motion. The deltoid plane was repaired followed by skin closure. Postoperatively, the operated arm was placed in a shoulder immobilizer. The patients were discharged the day after the surgery. Follow up visits were at one week, two weeks, six weeks, three months and one year.

The rehabilitation protocol consisted of simple range of motion exercises which were taught to the patients and relatives. They were encouraged to demonstrate the exercises so as to see whether they could replicate them. They were encouraged to repeat the exercises four times a day with ten repetitions at every time. These were started on the day of discharge. The elbow, forearm and hand exercises began on day one. Assisted shoulder forward flexion was started at one week. The patient holds both hands together and lifted their arms in front of their body bringing the shoulder in forward flexion. This was allowed as per pain tolerance. The patients were encouraged to increase the range as per pain tolerance. All patients started light use of operated hand at two weeks. Passive external rotation with elbow supported at the side of the body was started at two weeks. The technique of this manoeuvre was taught to the patient and relatives at the week two follow up. The patients used the shoulder immobilizer for six weeks. At six weeks, shoulder immobilizer was continued only at night and active shoulder motion including abduction and external rotation was started. Immobilizer was discontinued at two months. Weight lifting was allowed at this time in a gradually increasing manner. Overhead activities were started at three months.

At one year, we measured pain levels based on Visual analogue scale (VAS) score. Functional status was evaluated 
with Disabilities of the arm, shoulder and hand (DASH) score. Return to full work was noted. Range of motion and cuff strength were measured. Failure was defined as motion less than 50 percent of the opposite side or if some intervention was needed to improve the result.

\section{Results}

Mean duration of follow up was 14 months (range, 12 to 21 ). VAS score improved from preop mean 7.5 (range, 5 to 8 ) to postop mean 1.3 (range, 0 to 6 ). DASH was measured in 26 patients. In these 26 patients, DASH improved from preop mean 33.2 (range, 10.8 to 56.0) to follow up mean 4.5 (range, 0.8 to 11.0). Range of motion improved in 39 patients. Active abduction improved from preop mean 98 degrees (range, 40 to 130 ) to postop mean 134 degrees (range, 90 to 150). Forward flexion measured at 90 degrees of scapular plane improved from preop mean 98 degrees (range, 40 to 130 ) to postop mean 135 degrees (range, 90 to 150 ). External rotation measured with elbow next to the body and flexed at 90 degrees improved from preop mean 9 degrees (range 0 to 30 ) to postop mean 30 degrees (range, 0 to 50). Cuff strength was normal in 39 patients.

There were three failures (7\%). One patient developed significant stiffness in the postoperative period. He refused any intervention and continued with the protocol. He had less than 50 percent of motion as compared to opposite side at one year followup and had moderate pain (VAS of five). Two patients developed retear of the repaired cuff. They had persistent mild to moderate pain in postoperative period. MRI at three months showed a tear at the repaired tendon site. They were offered revision surgery but refused. They were lost to followup at about six months postop. Both these patients had large retracted $(>3 \mathrm{cms}$ ) tears and the repair was done under tension.

37 patients returned to full work by three months. The two patients with retears were unable to resume their work with the affected arm at the time of last followup. One failed patient who had developed stiffness and two patients with good result had changed their profession.

\section{Discussion}

Patient driven rehabilitation protocols following rotator cuff repairs can give good results in carefully selected patients. $[3,4]$ Patients can achieve over 90 percent of the range of motion. The present study showed improvement in DASH score and VAS score. 88 percent of the patients returned to previous work by three months of surgery. Failure was seen in three patients. This probably was the result of a repair done under tension. These were large tears and may have benefitted with a robust repair and possibly supervised therapy so as to not load the repair site. Other patients however did show successful return to work and improvement in their range of motion. These patients had good stable repairs following a tear repaired without tension.

Baumgarten et al in their systematic review concluded that there is not enough evidence to suggest ideal rehabilitation for rotator cuff surgery. [7]The included studies did not show any advantage of supervised protocol over unsupervised one, though the studies had weak designs. Some authors have shown better results with supervised programs in shoulder rehabilitation. Holmgren et al in a randomized controlled trial showed improved results with supervised program with progressive strengthening exercises and scapular stabilizers after arthroscopic acromioplasty when compared with home based program. [8] They showed improved functional score but the pain scores were not significantly different. Also they did not compare the costs involved.

Other studies have shown no difference between the two methods of rehabilitation. Andersen et al in a controlled clinical study of 43 patients did not find any significant differences. [9] These differences were not significant even in comparative studies for cuff repairs. $[10,11]$ Song et al found better SANE scores but similar range of motion and pain relief when comparing supervised and home therapy. [12] The rehabilitation began after four weeks of immobilization. The present study had a rehabilitation protocol where the exercises were begun at one week from surgery. Longo et al in their systematic review and metanalysis showed no difference between supervised versus unsupervised rehabilitation after rotator cuff repair in regards to the VAS scores and outcomes. [11]

Buker et al studied outcomes between supervised exercise program $(\mathrm{n}=15)$ and standardized home based program $(\mathrm{n}=13)$. [5] All patients underwent arthroscopic cuff repair. They found no difference in the pain score, Constant score and the functional status between the two groups. The study found supervised therapy program having higher costs. They initiated pendulum exercises in the initial six weeks period in both groups. The present study started with more exercises within the first six weeks.

The current study allowed patients to learn the rehabilitation protocol and perform it at home. This was started within a week from surgery. Gallagher et all have showed no difference between early and delayed rehabilitation after cuff repairs. [13] They do caution against early rehab in massive tears. The failures in the current group were also seen in patients with large tears with tension repair site.

One disadvantage of the home based unsupervised rehab protocol is that there is a risk that patient may end up doing too much and inadvertently cause damage to the repaired tendon. This can be avoided by carefully selecting patients for a homebased protocol. Smaller tears with a good stable repair will allow good 
rehabilitation. A massive tear with a difficult repair will benefit with a supervised program. In the present study, two of the three failures had large tears and repair was under tension. They would have benefited with a better repair and supervised therapy. Second disadvantage may be if the patient does too less. This can lead to stiffness and compromised function. Carefully following up with the patient and evaluating the progress can avoid this complication.

The study has its limitations. There is no control group where a similar group was treated with supervised protocol. There is also no control over the duration and the method in which the patients would follow the home based program.

In summary, home based exercise protocol for postoperative rotator cuff repairs can achieve good results with carefully selected patients. This will help in saving costs and time. Stable repair configuration in small tears and in those where there is not much retraction can benefit with home based protocols. We do not recommend this unsupervised home based rehabilitation in patients with large or retracted tears or in those whose repair may be done under tension.

\section{Clinical relevance}

The study describes a simple home based rehabilitation protocol after a rotator cuff repair. The study shows good results, pain relief and improved range of motion with the protocol. The patient does not have to go anywhere for the rehabilitation. This reduces the costs and time spent in travel. In the current pandemic, this also reduces the patient's risk of exposure.

Declaration of patient consent: The authors certify that they have obtained all appropriate patient consent forms. In the form, the patient has given his consent for his images and other clinical information to be reported in the Journal. The patient understands that his name and initials will not be published, and due efforts will be made to conceal his identity, but anonymity cannot be guaranteed.

Conflict of Interest: NIL; Source of Support: NIL

\section{References}

1. Beaudreuil J, Dhénain M, Coudane H, Mlika-Cabanne N. Clinical practice guidelines for the surgical management of rotator cuff tears in adults. Orthopaedics \& Traumatology: Surgery \& Research 2010; 96: 175-179.

2. Huisstede BM, Koes BW, Gebremariam L, Keijsers E, Verhaar JA. Current evidence for effectiveness of interventions to treat rotator cuff tears. Manual therapy 2011; 16: 217-230.

3. Hayes K, Ginn KA, Walton JR, Szomor ZL, Murrell GA. A randomised clinical trial evaluating the efficacy of physiotherapy after rotator cuff repair. Aust J Physiother 2004; 50:77-83.

4. Roddey TS, Olson SL, Gartsman GM, Hanten WP, Cook KF. A randomized controlled trial comparing 2 instructional approaches to home exercise instruction following arthroscopic full-thickness rotator cuff repair surgery. J Orthop Sports Phys Ther 2002; 32: 548-559.

5. Büker NA, Akkaya S, Akkaya N. Comparison of the results of supervised physiotherapy program and home-based exercise program in patients treated with arthroscopic-assisted miniopen rotator cuff repair. Eklem Hastalik Cerrahisi, 2011; 22(3): 134-139.

6. Lee BG, Cho NS, Rhee YG. Effect of two rehabilitation protocols on range of motion and healing rates after arthroscopic rotator cuff repair: aggressive versus limited early passive exercises. Arthroscopy 2012; 28(1): 34-42.

7. Baumgarten KM, Vidal AF, Wright RW. Rotator cuff rehabilitation. A level 1 and level 2 systematic review. Sports Health 2009; 1(2): 125-130.
8. Holmgren T, Oberg B, Sjöberg I, Johansson K. Supervised strengthening exercises versus home-based movement exercises after arthroscopic acromioplasty: a randomized clinical trial. J Rehabil Med 2012; 44(1): 12-18.

9. Andersen NH, Sojbjerg JO, Johannsen HV, Sneppen O. Selftraining versus physiotherapist-supervised rehabilitation of the shoulder in pa- tients treated with arthroscopic subacromial decompression: a clinical randomized study. J Shoulder Elbow Surg 1999; 8: 99-101.

10. Lisinski P, Huber J, Wilkosz P, Witkowska A, Wytrazek $M$, Samborski $W$ et al. Supervised versus uncontrolled rehabilitation of patients after rotator cuff repair-clinical and neurophysiological comparative study. Int. J Artif Organs 2012; 35: $45-54$.

11. Longo UG, Berton A, Ambrogioni LR, Lo Presti D, Carnevale A, Candela $V$ et al. Cost-Effectiveness of Supervised versus Unsupervised Rehabilitation for Rotator-Cuff Repair: Systematic Review and Meta-Analysis. Int J Environ Res Public Health 2020; 17: 2852

12. Song SJ, Jeong TH, Moon JW, Park HV, Lee SY, Koh KH. Short-term Comparison of Supervised Rehabilitation and Home- based Rehabilitation for Earlier Recovery of Shoulder Motion, Pain, and Function after Rotator Cuff Repair. Clinics in Shoulder and Elbow 2018; 21(1): 15-21.

13. Gallagher BP, Bishop ME, Tjoumakaris FP, Freedman KB. Early versus delayed rehabilitation following arthroscopic rotator cuff repair: A systematic review. Phys Sportsmed 2015; 43(2): 178-187.
Conflict of Interest: NIL Source of Support: NIL

\section{How to Cite this Article}

Habbu RA. Home based unsupervised rehabilitation protocol following rotator cuff repair has good outcomes: A prospective study. Journal of Clinical Orthopaedics July-Dec 2021;6(2):12-15. 